Bull. Korean Math. Soc. 50 (2013), No. 5, pp. 1567-1586

http://dx.doi.org/10.4134/BKMS.2013.50.5.1567

\title{
CURVATURE OF MULTIPLY WARPED PRODUCTS WITH AN AFFINE CONNECTION
}

\author{
YONG WANG
}

\begin{abstract}
In this paper, we study the Einstein multiply warped products with a semi-symmetric non-metric connection and the multiply warped products with a semi-symmetric non-metric connection with constant scalar curvature, we apply our results to generalized Robertson-Walker spacetimes with a semi-symmetric non-metric connection and generalized Kasner spacetimes with a semi-symmetric non-metric connection and find some new examples of Einstein affine manifolds and affine manifolds with constant scalar curvature. We also consider the multiply warped products with an affine connection with a zero torsion.
\end{abstract}

\section{Introduction}

The (singly) warped product $B \times{ }_{b} F$ of two pseudo-Riemannian manifolds $\left(B, g_{B}\right)$ and $\left(F, g_{F}\right)$ with a smooth function $b: B \rightarrow(0, \infty)$ is the product manifold $B \times F$ with the metric tensor $g=g_{B} \oplus b^{2} g_{F}$. Here, $\left(B, g_{B}\right)$ is called the base manifold and $\left(F, g_{F}\right)$ is called as the fiber manifold and $b$ is called as the warping function. Generalized Robertson-Walker space-times and standard static space-times are two well-known warped product spaces. The concept of warped products was first introduced by Bishop and Oneil (see [4]) to construct examples of Riemannian manifolds with negative curvature. In Riemannian geometry, warped product manifolds and their generic forms have been used to construct new examples with interesting curvature properties since then. In [5], F. Dobarro and E. Dozo had studied from the viewpoint of partial differential equations and variational methods, the problem of showing when a Riemannian metric of constant scalar curvature can be produced on a product manifolds by a warped product construction. In [7], Ehrlich, Jung and Kim got explicit solutions to warping function to have a constant scalar curvature for generalized Robertson-Walker space-times. In [3], explicit solutions were also obtained for the warping function to make the space-time as Einstein when the fiber is also Einstein.

Received August 17, 2012.

2010 Mathematics Subject Classification. 53C21, 53C50.

Key words and phrases. multiply warped products, semi-symmetric non-metric connection, Ricci tensor, scalar curvature, Einstein manifolds. 
One can generalize singly warped products to multiply warped products. Briefly, a multiply warped product $(M, g)$ is a product manifold of form $M=$ $B \times{ }_{b_{1}} F_{1} \times_{b_{2}} F_{2} \times \cdots \times_{b_{m}} F_{m}$ with the metric $g=g_{B} \oplus b_{1}^{2} g_{F_{1}} \oplus b_{2}^{2} g_{F_{2}} \oplus \cdots \oplus b_{m}^{2} g_{F_{m}}$, where for each $i \in\{1, \ldots, m\}, b_{i}: B \rightarrow(0, \infty)$ is smooth and $\left(F_{i}, g_{F_{i}}\right)$ is a pseudo-Riemannian manifold. In particular, when $B=(c, d)$ with the negative definite metric $g_{B}=-d t^{2}$ and $\left(F_{i}, g_{F_{i}}\right)$ is a Riemannian manifold, we call $M$ as the multiply generalized Robertson-Walker space-time. In [6], Dobarro and Ünal studied Ricci-flat and Einstein-Lorentzian multiply warped products and considered the case of having constant scalar curvature for multiply warped products and applied their results to generalized Kasner space-times.

Singly warped products have a natural generalization. A twisted product $(M, g)$ is a product manifold of form $M=B \times_{b} F$, with a smooth function $b: B \times F \rightarrow(0, \infty)$, and the metric tensor $g=g_{B} \oplus b^{2} g_{F}$. In [8], they showed that mixed Ricci-flat twisted products could be expressed as warped products. As a consequence, any Einstein twisted products are warped products. In [12], we define the multiply twisted products as generalizations of multiply warped products and twisted products. A multiply twisted product $(M, g)$ is a product manifold of form $M=B \times{ }_{b_{1}} F_{1} \times_{b_{2}} F_{2} \times \cdots \times \times_{b_{m}} F_{m}$ with the metric $g=g_{B} \oplus b_{1}^{2} g_{F_{1}} \oplus b_{2}^{2} g_{F_{2}} \oplus \cdots \oplus b_{m}^{2} g_{F_{m}}$, where for each $i \in\{1, \ldots, m\}, b_{i}: B \times F_{i} \rightarrow$ $(0, \infty)$ is smooth.

The definition of a semi-symmetric metric connection was given by H. Hayden in [9]. In 1970, K. Yano [13] considered a semi-symmetric metric connection and studied some of its properties. He proved that a Riemannian manifold admitting the semi-symmetric metric connection has vanishing curvature tensor if and only if it is conformally flat. Motivated by the Yano' result, in [11], Sular and Özgur studied warped product manifolds with a semi-symmetric metric connection, they computed curvature of semi-symmetric metric connection and considered Einstein warped product manifolds with a semi-symmetric metric connection. In [12], we considered multiply twisted products with a semisymmetric metric connection and computed the curvature of a semi-symmetric metric connection. We showed that mixed Ricci-flat multiply twisted products with a semi-symmetric metric connection can be expressed as multiply warped products which generalizes the result in [8]. We also studied the Einstein multiply warped products with a semi-symmetric metric connection and multiply warped products with a semi-symmetric metric connection with constant scalar curvature, we applied our results to generalized Robertson-Walker spacetimes with a semi-symmetric metric connection and generalized Kasner spacetimes with a semi-symmetric metric connection and we found some new examples of Einstein affine manifolds and affine manifolds with constant scalar curvature. We also classified generalized Einstein Robertson-Walker spacetimes with a semi-symmetric metric connection and generalized Einstein Kasner spacetimes with a semi-symmetric metric connection. In [1, 2], Agashe and Chafle introduced the notation of a semi-symmetric metric connection and studied some of 
its properties and submanifolds of a Riemannian manifold with semi-symmetric non-metric connections. In [10], they studied warped product manifolds with a semi-symmetric non-metric connection. The purpose of this paper is to study the Einstein multiply warped products with a semi-symmetric non-metric connection and multiply warped products with a semi-symmetric non-metric connection with constant scalar curvature.

This paper is arranged as follows: In Section 2, we compute curvature of multiply twisted products with a semi-symmetric non-metric connection. In Section 3 , we study the special multiply warped products with a semi-symmetric non-metric connection. In Section 4, we study the generalized RobertsonWalker spacetimes with a semi-symmetric non-metric connection. In Section 5, we consider the generalized Kasner spacetimes with a semi-symmetric nonmetric connection. In Section 6, we compute curvature of multiply twisted products with an affine connection with a zero torsion.

\section{Preliminaries}

Definition 2.1. A multiply twisted product $(M, g)$ is a product manifold of form $M=B \times_{b_{1}} F_{1} \times_{b_{2}} F_{2} \times \cdots \times_{b_{m}} F_{m}$ with the metric $g=g_{B} \oplus b_{1}^{2} g_{F_{1}} \oplus$ $b_{2}^{2} g_{F_{2}} \oplus \cdots \oplus b_{m}^{2} g_{F_{m}}$, where for each $i \in\{1, \ldots, m\}, b_{i}: B \times F_{i} \rightarrow(0, \infty)$ is smooth.

Here, $\left(B, g_{B}\right)$ is called the base manifold and $\left(F_{i}, g_{F_{i}}\right)$ is called as the fiber manifold and $b_{i}$ is called as the twisted function. Obviously, twisted products and multiply warped products are the special cases of multiply twisted products.

Let $M$ be a Riemannian manifold with Riemannian metric $g$. A linear connection $\bar{\nabla}$ on a Riemannian manifold $M$ is called a semi-symmetric connection if the torsion tensor $T$ of the connection $\bar{\nabla}$

$$
T(X, Y)=\bar{\nabla}_{X} Y-\bar{\nabla}_{Y} X-[X, Y]
$$

satisfies

$$
T(X, Y)=\pi(Y) X-\pi(X) Y,
$$

where $\pi$ is a 1-form associated with the vector field $P$ on $M$ defined by $\pi(X)=$ $g(X . P) . \bar{\nabla}$ is called a semi-symmetric metric connection if it satisfies $\bar{\nabla} g=0$. $\bar{\nabla}$ is called a semi-symmetric non-metric connection if it satisfies $\bar{\nabla} g \neq 0$.

If $\nabla$ is the Levi-Civita connection of $M$, the semi-symmetric non-metric connection $\bar{\nabla}$ is given by

$$
\bar{\nabla}_{X} Y=\nabla_{X} Y+\pi(Y) X
$$

(see $[1]$ ). Let $R$ and $\bar{R}$ be the curvature tensors of $\nabla$ and $\bar{\nabla}$, respectively. Then $R$ and $\bar{R}$ are related by

$$
\begin{aligned}
\bar{R}(X, Y) Z= & R(X, Y) Z+g\left(Z, \nabla_{X} P\right) Y-g\left(Z, \nabla_{Y} P\right) X \\
& +\pi(Z)[\pi(Y) X-\pi(X) Y]
\end{aligned}
$$


for any vector fields $X, Y, Z$ on $M[1]$. By (2.3) and Proposition 2.2 in [12], we have:

Proposition 2.2. Let $M=B \times_{b_{1}} F_{1} \times_{b_{2}} F_{2} \times \cdots \times_{b_{m}} F_{m}$ be a multiply twisted product and let $X, Y \in \Gamma(T B)$ and $U \in \Gamma\left(T F_{i}\right), W \in \Gamma\left(T F_{j}\right)$ and $P \in \Gamma(T B)$. Then

(1) $\bar{\nabla}_{X} Y=\bar{\nabla}_{X}^{B} Y$.

(2) $\bar{\nabla}_{X} U=\frac{X\left(b_{i}\right)}{b_{i}} U$.

(3) $\bar{\nabla}_{U} X=\left[\frac{X\left(b_{i}\right)}{b_{i}}+\pi(X)\right] U$.

(4) $\bar{\nabla}_{U} W=0$ if $i \neq j$.

(5) $\bar{\nabla}_{U} W=U\left(\ln b_{i}\right) W+W\left(\ln b_{i}\right) U-\frac{g_{F_{i}}(U, W)}{b_{i}} \operatorname{grad}_{F_{i}} b_{i}-b_{i} g_{F_{i}}(U, W) \operatorname{grad}_{B} b_{i}$ $+\nabla_{U}^{F_{i}} W$ if $i=j$.

Proposition 2.3. Let $M=B \times_{b_{1}} F_{1} \times_{b_{2}} F_{2} \times \cdots \times_{b_{m}} F_{m}$ be a multiply twisted product and let $X, Y \in \Gamma(T B)$ and $U \in \Gamma\left(T F_{i}\right), W \in \Gamma\left(T F_{j}\right)$ and $P \in \Gamma\left(T F_{k}\right)$. Then

(1) $\bar{\nabla}_{X} Y=\nabla_{X}^{B} Y$.

(2) $\bar{\nabla}_{X} U=\frac{X\left(b_{i}\right)}{b_{i}} U+g(P, U) X$.

(3) $\bar{\nabla}_{U} X=\frac{X\left(b_{i}\right)}{b_{i}} U$.

(4) $\bar{\nabla}_{U} W=g(W, P) U$ if $i \neq j$.

(5) $\bar{\nabla}_{U} W=U\left(\ln b_{i}\right) W+W\left(\ln b_{i}\right) U-\frac{g_{F_{i}}(U, W)}{b_{i}} \operatorname{grad}_{F_{i}} b_{i}-b_{i} g_{F_{i}}(U, W) \operatorname{grad}_{B} b_{i}$ $+\nabla_{U}^{F_{i}} W+\pi(W) U$ if $i=j$.

By (2.4) and Proposition 2.4 in [12], we have:

Proposition 2.4. Let $M=B \times_{b_{1}} F_{1} \times_{b_{2}} F_{2} \times \cdots \times_{b_{m}} F_{m}$ be a multiply twisted product and let $X, Y, Z \in \Gamma(T B)$ and $V \in \Gamma\left(T F_{i}\right), W \in \Gamma\left(T F_{j}\right), U \in \Gamma\left(T F_{k}\right)$ and $P \in \Gamma(T B)$. Then

(1) $\bar{R}(X, Y) Z=\bar{R}^{B}(X, Y) Z$.

(2) $\bar{R}(V, X) Y=-\left[\frac{H_{B}^{b_{i}}(X, Y)}{b_{i}}+g\left(Y, \nabla_{X} P\right)-\pi(X) \pi(Y)\right] V$.

(3) $\bar{R}(X, V) W=\bar{R}(V, W) X=\bar{R}(V, X) W=0$ if $i \neq j$.

(4) $\bar{R}(X, Y) V=0$.

(5) $\bar{R}(V, W) X=V X\left(\ln b_{i}\right) W-W X\left(\ln b_{i}\right) V$ if $i=j$.

(6) $\bar{R}(V, W) U=0$ if $i=j \neq k$ or $i \neq j \neq k$.

(7) $\bar{R}(U, V) W=-g(V, W) \frac{g_{B}\left(\operatorname{grad}_{B} b_{i}, \operatorname{grad}_{B} b_{k}\right)}{b_{i} b_{k}} U-g(V, W) \frac{P\left(b_{i}\right)}{b_{i}} U$ if $i=j \neq k$.

(8) $\bar{R}(X, V) W=\left[W X\left(\ln b_{i}\right)\right] V-g(W, V) \cdot\left[\frac{\nabla_{X}^{B}\left(\operatorname{grad}_{B} b_{i}\right)}{b_{i}}+\frac{\operatorname{grad}_{F_{i}}\left(X \ln b_{i}\right)}{b_{i}^{2}}\right.$ $\left.+\frac{P\left(b_{i}\right)}{b_{i}} X\right]$ if $i=j$.

(9) $\bar{R}(U, V) W=g(U, W) \operatorname{grad}_{B}\left(V\left(\ln b_{i}\right)\right)-g(V, W) \operatorname{grad}_{B}\left(U\left(\ln b_{i}\right)\right)$ $+R^{F_{i}}(U, V) W$ 


$$
\begin{aligned}
& -\left(\frac{\left.\operatorname{grad}_{B} b_{i}\right|_{B} ^{2}}{b_{i}^{2}}+\frac{P\left(b_{i}\right)}{b_{i}}\right)[g(V, W) U-g(U, W) V] \\
& \text { if } i=j=k .
\end{aligned}
$$

Proposition 2.5. Let $M=B \times_{b_{1}} F_{1} \times_{b_{2}} F_{2} \times \cdots \times_{b_{m}} F_{m}$ be a multiply twisted product and let $X, Y, Z \in \Gamma(T B)$ and $V \in \Gamma\left(T F_{i}\right), W \in \Gamma\left(T F_{j}\right), U \in \Gamma\left(T F_{k}\right)$ and $P \in \Gamma\left(T F_{l}\right)$. Then

(1) $\bar{R}(X, Y) Z=R^{B}(X, Y) Z$.

(2) $\bar{R}(V, X) Y=-\frac{H_{B}^{b_{i}}(X, Y)}{b_{i}} V$ if $i \neq l$.

(3) $\bar{R}(V, X) Y=-\frac{H_{B}^{b_{i}}(X, Y)}{b_{i}} V-\pi(V) \frac{Y\left(b_{i}\right)}{b_{i}} X$ if $i=l$.

(4) $\bar{R}(X, V) W=\frac{X\left(b_{l}\right)}{b_{l}} \pi(W) V$ if $i \neq j$.

(5) $\bar{R}(V, W) X=-\delta_{i}^{l} \frac{\pi(V)}{b_{i}} X\left(b_{i}\right) W+\delta_{j}^{l} \frac{\pi(W)}{b_{j}} X\left(b_{j}\right) V$ if $i \neq j$.

(6) $\bar{R}(X, Y) V=\pi(V)\left[\frac{X\left(b_{l}\right)}{b_{l}} Y-\frac{Y\left(b_{l}\right)}{b_{l}} X\right]$.

(7) $\bar{R}(V, W) X=V X\left(\ln b_{i}\right) W-W X\left(\ln b_{i}\right) V-\delta_{i}^{l} \frac{X\left(b_{i}\right)}{b_{i}}[\pi(V) W-\pi(W) V]$ if $i=j$.

(8) $\bar{R}(V, W) U=0$ if $i=j \neq k$ or $i \neq j \neq k$.

(9) $\bar{R}(U, V) W=-g(V, W) \frac{g_{B}\left(\operatorname{grad}_{B} b_{i}, \operatorname{grad}_{B} b_{k}\right)}{b_{i} b_{k}} U-g\left(W, \nabla_{V} P\right) U$ $+\pi(W)[\pi(V) U-\pi(U) V]$ if $i=j \neq k$.

(10) $\bar{R}(X, V) W=\left[W X\left(\ln b_{i}\right)\right] V-g(W, V) \frac{\nabla_{X}^{B}\left(\operatorname{grad}_{B} b_{i}\right)}{b_{i}}$ $-\operatorname{grad}_{F_{i}}\left(X \ln b_{i}\right) g_{F_{i}}(W, V)+\frac{X\left(b_{l}\right)}{b_{l}} \pi(W) V$ $-g\left(W, \nabla_{V} P\right) X+\pi(V) \pi(W) X$ if $i=j$.

(11) $\bar{R}(U, V) W=g(U, W) \operatorname{grad}_{B}\left(V\left(\ln b_{i}\right)\right)-g(V, W) \operatorname{grad}_{B}\left(U\left(\ln b_{i}\right)\right)$

$$
\begin{aligned}
& +R^{F_{i}}(U, V) W-\frac{\left|\operatorname{grad}_{B} b_{i}\right|_{B}^{2}}{b_{i}^{2}}(g(V, W) U-g(U, W) V) \\
& \text { if } i=j=k \neq l .
\end{aligned}
$$

(12) $\bar{R}(U, V) W=g(U, W) \operatorname{grad}_{B}\left(V\left(\ln b_{i}\right)\right)-g(V, W) \operatorname{grad}_{B}\left(U\left(\ln b_{i}\right)\right)$

$$
\begin{aligned}
& +R^{F_{i}}(U, V) W-\frac{\left.\operatorname{grad}_{B} b_{i}\right|_{B} ^{2}}{b_{i}^{2}}(g(V, W) U-g(U, W) V) \\
& +g\left(W, \nabla_{U} P\right) V-g\left(W, \nabla_{V} P\right) U+\pi(W)[\pi(V) U-\pi(U) V] \\
& \text { if } i=j=k=l .
\end{aligned}
$$

By Propositions 2.4 and 2.5, we have:

Proposition 2.6. Let $M=B \times_{b_{1}} F_{1} \times_{b_{2}} F_{2} \times \cdots \times_{b_{m}} F_{m}$ be a multiply twisted product and let $X, Y, Z \in \Gamma(T B)$ and $V \in \Gamma\left(T F_{i}\right), W \in \Gamma\left(T F_{j}\right)$ and $P \in$ $\Gamma(T B)$. Then

(1) $\overline{\operatorname{Ric}}(X, Y)=\overline{\operatorname{Ric}}^{B}(X, Y)+\sum_{i=1}^{m} l_{i}\left[\frac{H_{B}^{b_{i}}(X, Y)}{b_{i}}+g\left(Y, \nabla_{X} P\right)-\pi(X) \pi(Y)\right]$.

(2) $\overline{\operatorname{Ric}}(X, V)=\overline{\operatorname{Ric}}(V, X)=\left(l_{i}-1\right)\left[V X\left(\ln b_{i}\right)\right]$.

(3) $\overline{\operatorname{Ric}}(V, W)=0$ if $i \neq j$.

(4) $\overline{\operatorname{Ric}}(V, W)=\operatorname{Ric}^{F_{i}}(V, W)+\left[\frac{\triangle_{B} b_{i}}{b_{i}}+\left(l_{i}-1\right) \frac{\left.\operatorname{|grad}_{B} b_{i}\right|_{B} ^{2}}{b_{i}^{2}}\right.$

$\left.+\sum_{j \neq i} l_{j} \frac{g_{B}\left(\operatorname{grad}_{B} b_{i}, \operatorname{grad}_{B} b_{j}\right)}{b_{i} b_{j}}+\sum_{j=1}^{m} l_{j} \frac{P b_{j}}{b_{j}}+(n-1) \frac{P b_{i}}{b_{i}}\right] g(V, W)$ if $i=$ $j$, where $\operatorname{dim} B=n, \operatorname{dim} M=\bar{n}$. 
Corollary 2.7. Let $M=B \times_{b_{1}} F_{1} \times_{b_{2}} F_{2} \times \cdots \times_{b_{m}} F_{m}$ be a multiply twisted product and $\operatorname{dim} F_{i}>1$ and $P \in \Gamma(T B)$. Then $(M, \bar{\nabla})$ is mixed Ricci-flat if and only if $M$ can be expressed as a multiply warped product. In particular, if $(M, \bar{\nabla})$ is Einstein, then $M$ can be expressed as a multiply warped product.

Proposition 2.8. Let $M=B \times_{b_{1}} F_{1} \times_{b_{2}} F_{2} \times \cdots \times_{b_{m}} F_{m}$ be a multiply twisted product and let $X, Y, Z \in \Gamma(T B)$ and $V \in \Gamma\left(T F_{i}\right), W \in \Gamma\left(T F_{j}\right)$ and $P \in$ $\Gamma\left(T F_{r}\right)$. Then

(1) $\overline{\operatorname{Ric}}(X, Y)=\operatorname{Ric}^{B}(X, Y)+\sum_{i=1}^{m} l_{i} \frac{H_{B}^{b_{i}}(X, Y)}{b_{i}}$.

(2) $\overline{\operatorname{Ric}}(X, V)=\left(l_{i}-1\right)\left[V X\left(\ln b_{i}\right)\right]+(\bar{n}-1) \frac{X\left(b_{r}\right)}{b_{r}} \pi(V)$.

(3) $\overline{\operatorname{Ric}}(V, X)=\left(l_{i}-1\right)\left[V X\left(\ln b_{i}\right)\right]+(1-\bar{n}) \frac{X\left(b_{r}\right)}{b_{r}} \pi(V)$.

(4) $\overline{\operatorname{Ric}}(V, W)=0$ if $i \neq j$.

(5) $\overline{\operatorname{Ric}}(V, W)=\operatorname{Ric}^{F_{i}}(V, W)+g(V, W)\left[\frac{\triangle_{B} b_{i}}{b_{i}}+\left(l_{i}-1\right) \frac{\left.\operatorname{grad}_{B} b_{i}\right|_{B} ^{2}}{b_{i}^{2}}\right.$

$\left.+\sum_{j \neq i} l_{j} \frac{g_{B}\left(\operatorname{grad}_{B} b_{i}, \operatorname{grad}_{B} b_{j}\right)}{b_{i} b_{j}}\right]+(\bar{n}-1) g\left(W, \nabla_{V} P\right)+(1-\bar{n}) \pi(V) \pi(W)$ if $i=j$.

Corollary 2.9. Let $M=B \times_{b_{1}} F_{1} \times_{b_{2}} F_{2} \times \cdots \times_{b_{m}} F_{m}$ be a multiply twisted product and $\operatorname{dim} F_{i}>1$ and $P \in \Gamma\left(T F_{r}\right)$. Then $(M, \bar{\nabla})$ is mixed Ricci-flat if and only if $M$ can be expressed as a multiply warped product and $b_{r}$ is only dependent on $F_{r}$. In particular, if $(M, \bar{\nabla})$ is Einstein, then $M$ can be expressed as a multiply warped product.

Proposition 2.10. Let $M=B \times_{b_{1}} F_{1} \times_{b_{2}} F_{2} \times \cdots \times_{b_{m}} F_{m}$ be a multiply twisted product and $P \in \Gamma(T B)$. Then the scalar curvature $\bar{S}$ has the following expression:

$$
\begin{aligned}
\bar{S}= & \bar{S}^{B}+2 \sum_{i=1}^{m} \frac{l_{i}}{b_{i}} \triangle_{B} b_{i}+\sum_{i=1}^{m} \frac{S^{F_{i}}}{b_{i}^{2}}+\sum_{i=1}^{m} l_{i}\left(l_{i}-1\right) \frac{\left|\operatorname{grad}_{B} b_{i}\right|_{B}^{2}}{b_{i}^{2}} \\
& +\sum_{i=1}^{m} \sum_{j \neq i} l_{i} l_{j} \frac{g_{B}\left(\operatorname{grad}_{B} b_{i}, \operatorname{grad}_{B} b_{j}\right)}{b_{i} b_{j}}+(n-1) \sum_{i=1}^{m} l_{i} \frac{P\left(b_{i}\right)}{b_{i}} \\
& +\sum_{i=1}^{m} \sum_{j=1}^{m} l_{i} l_{j} \frac{P\left(b_{j}\right)}{b_{j}}+\sum_{i=1}^{m} l_{i}\left[\operatorname{div}_{B} P-\pi(P)\right] .
\end{aligned}
$$

Proposition 2.11. Let $M=B \times_{b_{1}} F_{1} \times_{b_{2}} F_{2} \times \cdots \times_{b_{m}} F_{m}$ be a multiply twisted product and $P \in \Gamma\left(T F_{r}\right)$. Then the scalar curvature $\bar{S}$ has the following expression:

$$
\begin{aligned}
\bar{S}= & S^{B}+2 \sum_{i=1}^{m} \frac{l_{i}}{b_{i}} \triangle_{B} b_{i}+\sum_{i=1}^{m} \frac{S^{F_{i}}}{b_{i}^{2}}+\sum_{i=1}^{m} l_{i}\left(l_{i}-1\right) \frac{\left|\operatorname{grad}_{B} b_{i}\right|_{B}^{2}}{b_{i}^{2}} \\
& +\sum_{i=1}^{m} \sum_{j \neq i} l_{i} l_{j} \frac{g_{B}\left(\operatorname{grad}_{B} b_{i}, \operatorname{grad}_{B} b_{j}\right)}{b_{i} b_{j}}+(1-\bar{n}) \pi(P)
\end{aligned}
$$




$$
+(\bar{n}-1) \sum_{j_{r}=1}^{l_{r}} \varepsilon_{j_{r}} g\left(\nabla_{E_{j_{r}}^{r}} P, E_{j_{r}}^{r}\right) .
$$

\section{Special multiply warped products with a semi-symmetric non-metric connection}

Let $M=I \times_{b_{1}} F_{1} \times_{b_{2}} F_{2} \times \cdots \times_{b_{m}} F_{m}$ be a multiply warped product with the metric tensor $-d t^{2} \oplus b_{1}^{2} g_{F_{1}} \oplus \cdots \oplus b_{m}^{2} g_{F_{m}}$ and $I$ is an open interval in $\mathbb{R}$ and $b_{i} \in C^{\infty}(I)$.

Theorem 3.1. Let $M=I \times_{b_{1}} F_{1} \times_{b_{2}} F_{2} \times \cdots \times_{b_{m}} F_{m}$ be a multiply warped product with the metric tensor $-d t^{2} \oplus b_{1}^{2} g_{F_{1}} \oplus \cdots \oplus b_{m}^{2} g_{F_{m}}$ and $P=\frac{\partial}{\partial t}$. Then $(M, \bar{\nabla})$ is Einstein with the Einstein constant $\lambda$ if and only if the following conditions are satisfied for any $i \in\{1, \ldots, m\}$

(1) $\left(F_{i}, \nabla^{F_{i}}\right)$ is Einstein with the Einstein constant $\lambda_{i}, i \in\{1, \ldots, m\}$.

(2) $\sum_{i=1}^{m} l_{i}\left(1-\frac{b_{i}^{\prime \prime}}{b_{i}}\right)=\lambda$.

(3) $\lambda_{i}-b_{i} b_{i}^{\prime \prime}-\left(l_{i}-1\right) b_{i}^{2}-b_{i} b_{i}^{\prime} \sum_{j \neq i} l_{j} \frac{b_{j}^{\prime}}{b_{j}}+b_{i}^{2} \sum_{j=1}^{m} l_{j} \frac{b_{j}^{\prime}}{b_{j}}=\lambda b_{i}^{2}$.

Proof. By Proposition 2.6, we have

$$
\begin{gathered}
\overline{\operatorname{Ric}}\left(\frac{\partial}{\partial t}, \frac{\partial}{\partial t}\right)=-\sum_{i=1}^{m} l_{i}\left(1-\frac{b_{i}^{\prime \prime}}{b_{i}}\right) ; \\
\overline{\operatorname{Ric}}\left(\frac{\partial}{\partial t}, V\right)=\overline{\operatorname{Ric}}\left(V, \frac{\partial}{\partial t}\right)=0 ;
\end{gathered}
$$

$$
\begin{aligned}
\overline{\operatorname{Ric}}(V, W)= & \operatorname{Ric}^{F_{i}}(V, W) \\
& +g_{F_{i}}(V, W)\left[-b_{i} b_{i}^{\prime \prime}-\left(l_{i}-1\right) b_{i}^{\prime 2}-b_{i} b_{i}^{\prime} \sum_{j \neq i} l_{j} \frac{b_{j}^{\prime}}{b_{j}}+b_{i}^{2} \sum_{j=1}^{m} l_{j} \frac{b_{j}^{\prime}}{b_{j}}\right] .
\end{aligned}
$$

By (3.1)-(3.3) and the Einstein condition, we get the above theorem.

Definition 3.2. $(M, \bar{\nabla})$ is called pseudo-Einstein with the Einstein constant $\lambda$ if $\frac{1}{2}[\overline{\operatorname{Ric}}(X, Y)+\overline{\operatorname{Ric}}(Y, X)]=\lambda g(X, Y)$.

Theorem 3.3. Let $M=I \times_{b_{1}} F_{1} \times_{b_{2}} F_{2} \times \cdots \times_{b_{m}} F_{m}$ be a multiply warped product with the metric tensor $-d t^{2} \oplus b_{1}^{2} g_{F_{1}} \oplus \cdots \oplus b_{m}^{2} g_{F_{m}}$ and $P \in \Gamma\left(T F_{r}\right)$ and $\bar{n}>2$. Then $(M, \bar{\nabla})$ is pseudo-Einstein with the Einstein constant $\lambda$ if and only if the following conditions are satisfied for any $i \in\{1, \ldots, m\}$

(1) $\left(F_{i}, \nabla^{F_{i}}\right)(i \neq r)$ is Einstein with the Einstein constant $\lambda_{i}, i \in\{1, \ldots$, $m\}$.

(2) $-\sum_{i=1}^{m} l_{i} \frac{b_{i}^{\prime \prime}}{b_{i}}=\lambda$. 
(3) $\operatorname{Ric}^{F_{i}}(V, W)-g_{F_{i}}(V, W)\left[b_{i} b_{i}^{\prime \prime}+\left(l_{i}-1\right) b_{i}^{\prime 2}+b_{i} b_{i}^{\prime} \sum_{j \neq i} l_{j} \frac{b_{j}^{\prime}}{b_{j}}+\lambda b_{i}^{2}\right]=$ $(\bar{n}-1)\left[\pi(V) \pi(W)-\frac{g\left(W, \nabla_{V} P\right)+g\left(V, \nabla_{W} P\right)}{2}\right]$ for $V, W \in \Gamma\left(T F_{r}\right), r=i$.

(4) $\lambda_{i}-b_{i} b_{i}^{\prime \prime}-\left(l_{i}-1\right) b_{i}^{\prime 2}-b_{i} b_{i}^{\prime} \sum_{j \neq i} l_{j} \frac{b_{j}^{\prime}}{b_{j}}-\lambda b_{i}^{2}=0$ for $i \neq r$.

Proof. By Proposition 2.8, then,

$$
\overline{\operatorname{Ric}}\left(\frac{\partial}{\partial t}, \frac{\partial}{\partial t}\right)=\sum_{i=1}^{m} l_{i} \frac{b_{i}^{\prime \prime}}{b_{i}}
$$

So we have $\sum_{i=1}^{m} l_{i} \frac{b_{i}^{\prime \prime}}{b_{i}}=-\lambda$.

$$
\begin{aligned}
\overline{\operatorname{Ric}}(V, W)= & \operatorname{Ric}^{F_{i}}(V, W)+b_{i}^{2} g_{F_{i}}(V, W)\left[-\frac{b_{i}^{\prime \prime}}{b_{i}}+\left(l_{i}-1\right) \frac{-b_{i}^{\prime 2}}{b_{i}^{2}}+\sum_{j \neq i} l_{j} \frac{-b_{i}^{\prime} b_{j}^{\prime}}{b_{i} b_{j}}\right] \\
& +(\bar{n}-1)\left[g\left(W, \nabla_{V} P\right)-\pi(V) \pi(W)\right] .
\end{aligned}
$$

When $i \neq r$, then $\nabla_{V} P=\nabla_{W} P=\pi(V)=0$, so

$$
\begin{aligned}
\overline{\operatorname{Ric}}(V, W) & =\operatorname{Ric}^{F_{i}}(V, W)+b_{i}^{2} g_{F_{i}}(V, W)\left[-\frac{b_{i}^{\prime \prime}}{b_{i}}+\left(l_{i}-1\right) \frac{-b_{i}^{\prime 2}}{b_{i}^{2}}+\sum_{j \neq i} l_{j} \frac{-b_{i}^{\prime} b_{j}^{\prime}}{b_{i} b_{j}}\right] \\
& =\lambda b_{i}^{2} g_{F_{i}}(V, W) .
\end{aligned}
$$

By variables separation, we have $\left(F_{i}, \nabla^{F_{i}}\right)(i \neq r)$ is Einstein with the Einstein constant $\lambda_{i}$ and

$$
\lambda_{i}-b_{i} b_{i}^{\prime \prime}-b_{i} b_{i}^{\prime} \sum_{j \neq i} l_{j} \frac{b_{j}^{\prime}}{b_{j}}-\left(l_{i}-1\right) b_{i}^{2}=\lambda b_{i}^{2} .
$$

When $i=r$, then

$$
\begin{aligned}
& \operatorname{Ric}^{F_{i}}(V, W)-g_{F_{i}}(V, W)\left[b_{i} b_{i}^{\prime \prime}+\left(l_{i}-1\right) b_{i}^{\prime 2}+b_{i} b_{i}^{\prime} \sum_{j \neq i} l_{j} \frac{b_{j}^{\prime}}{b_{j}}+\lambda b_{i}^{2}\right] \\
= & (\bar{n}-1)\left[\pi(V) \pi(W)-\frac{g\left(W, \nabla_{V} P\right)+g\left(V, \nabla_{W} P\right)}{2}\right] .
\end{aligned}
$$

So we prove the above theorem.

When $M=I \times_{b_{1}} F_{1} \times_{b_{2}} F_{2} \times \cdots \times_{b_{m}} F_{m}$ be a multiply warped product and $P=\frac{\partial}{\partial t}$, by Proposition 2.10, we have

$\bar{S}=-2 \sum_{i=1}^{m} l_{i} \frac{b_{i}^{\prime \prime}}{b_{i}}+\sum_{i=1}^{m} \frac{S^{F_{i}}}{b_{i}^{2}}+\sum_{i=1}^{m} l_{i}-\sum_{i=1}^{m} l_{i}\left(l_{i}-1\right) \frac{b_{i}^{\prime 2}}{b_{i}^{2}}-\sum_{i=1}^{m} \sum_{j \neq i} l_{i} l_{j} \frac{b_{i}^{\prime} b_{j}^{\prime}}{b_{i} b_{j}}+\sum_{i, j=1}^{m} l_{i} l_{j} \frac{b_{j}^{\prime}}{b_{j}}$. 
The following result just follows from the method of separation of variables and the fact that each $S^{F_{i}}$ is a function defined on $F_{i}$.

Proposition 3.4. Let $M=I \times_{b_{1}} F_{1} \times_{b_{2}} F_{2} \times \cdots \times_{b_{m}} F_{m}$ be a multiply warped product and $P=\frac{\partial}{\partial t}$. If $(M, \bar{\nabla})$ has constant scalar curvature $\bar{S}$, then each $\left(F_{i}, \nabla^{F_{i}}\right)$ has constant scalar curvature $S^{F_{i}}$.

When $P \in \Gamma\left(T F_{r}\right)$, by Proposition 2.11, we have

$$
\begin{aligned}
\bar{S}= & -2 \sum_{i=1}^{m} l_{i} \frac{b_{i}^{\prime \prime}}{b_{i}}+\sum_{i=1}^{m} \frac{S^{F_{i}}}{b_{i}^{2}}+\sum_{i=1}^{m} l_{i}\left(l_{i}-1\right) \frac{-b_{i}^{\prime 2}}{b_{i}^{2}}+\sum_{i=1}^{m} \sum_{j \neq i} l_{i} l_{j} \frac{-b_{i}^{\prime} b_{j}^{\prime}}{b_{i} b_{j}} \\
& +\pi(P)(1-\bar{n})+(\bar{n}-1) \operatorname{div}_{F_{r}} P .
\end{aligned}
$$

Proposition 3.5. Let $M=I \times_{b_{1}} F_{1} \times_{b_{2}} F_{2} \times \cdots \times_{b_{m}} F_{m}$ be a multiply warped product and $P \in \Gamma\left(T F_{r}\right)$. If $(M, \bar{\nabla})$ has constant scalar curvature $\bar{S}$, then each $\left(F_{i}, \nabla^{F_{i}}\right)(i \neq r)$ has constant scalar curvature $S^{F_{i}}$ and if $g_{F_{r}}(P, P)$ and $\operatorname{div}_{F_{r}} P$ are constants, then $S^{F_{r}}$ is also a constant.

\section{Generalized Robertson-Walker spacetimes with a semi-symmetric non-metric connection}

In this section, we study $M=I \times F$ with the metric tensor $-d t^{2}+f(t)^{2} g_{F}$. As a corollary of Theorem 3.1, we obtain:

Corollary 4.1. Let $M=I \times F$ with the metric tensor $-d t^{2}+f(t)^{2} g_{F}$ and $P=\frac{\partial}{\partial t}$. Then $(M, \bar{\nabla})$ is Einstein with the Einstein constant $\lambda$ if and only if the following conditions are satisfied

(1) $\left(F, \nabla^{F}\right)$ is Einstein with the Einstein constant $\lambda_{F}$.

(2) $f^{\prime \prime}=\left(1-\frac{\lambda}{l}\right) f$.

(3) $\lambda_{F}+(1-l) f^{\prime 2}+\left(\frac{\lambda}{l}-1-\lambda\right) f^{2}+l f^{\prime} f=0$.

By Corollary 4.1(2) and elementary methods for ordinary differential equations, we get:

Case i) $\lambda<l$, then $f=c_{1} e^{a t}+c_{2} e^{-a t}$, where $a=\sqrt{1-\frac{\lambda}{l}}$. By Corollary 4.1 $(3)$, then

$$
\lambda_{F}+2 c_{1} c_{2}\left[\frac{\lambda}{l}-1-\lambda+(l-1) a^{2}\right]+c_{1}^{2} e^{2 a t}\left[(1-l) a^{2}+\left(\frac{\lambda}{l}-1-\lambda\right)+l a\right]
$$

$$
+c_{2}^{2} e^{-2 a t}\left[(1-l) a^{2}+\left(\frac{\lambda}{l}-1-\lambda\right)-l a\right]=0
$$

So

$$
\begin{gathered}
\lambda_{F}+2 c_{1} c_{2}\left[\frac{\lambda}{l}-1-\lambda+(l-1) a^{2}\right]=0 \\
c_{1}^{2}\left[(1-l) a^{2}+\left(\frac{\lambda}{l}-1-\lambda\right)+l a\right]=0
\end{gathered}
$$




$$
c_{2}^{2}\left[(1-l) a^{2}+\left(\frac{\lambda}{l}-1-\lambda\right)-l a\right]=0 .
$$

When $c_{1} \neq 0, c_{2} \neq 0$, by (4.2ii) and (4.2iii), then $l a=0$, this is a contradiction. When $c_{1}=0$ and $c_{2} \neq 0$, by (4.2iii), $(1-l) a^{2}+\left(\frac{\lambda}{l}-1-\lambda\right)=l a$. By $a=\sqrt{1-\frac{\lambda}{l}}$, then $a=-1$, this is a contradiction. When $c_{1} \neq 0$ and $c_{2}=0$, by (4.2ii), $(1-l) a^{2}+\left(\frac{\lambda}{l}-1-\lambda\right)=-l a$, then $a=1$ and $\lambda=0$. By (4.2i), $\lambda_{F}=0$. So we get $\underline{\lambda_{F}=\lambda=0, f=c_{1} e^{t}}$.

Case ii) $\lambda>l, f=c_{1} \cos (b t)+c_{2} \sin (b t)$, where $b=\sqrt{\frac{\lambda}{l}-1}$. By Corollary 4.1(3), we have

$$
\begin{aligned}
& \lambda_{F}+(1-l)\left(-c_{1} b \sin (b t)+c_{2} b \cos (b t)\right)^{2}+\left(\frac{\lambda}{l}-1-\lambda\right)\left(c_{1} \cos (b t)+c_{2} \sin (b t)\right)^{2} \\
& +l\left(c_{1} \cos (b t)+c_{2} \sin (b t)\right)\left(-c_{1} b \sin (b t)+c_{2} b \cos (b t)\right)=0 .
\end{aligned}
$$

So

$$
\begin{gathered}
(1-l) c_{1}^{2} b^{2}+\left(\frac{\lambda}{l}-1-\lambda\right) c_{2}^{2}-l c_{1} c_{2} b=-\lambda_{F} ; \\
(1-l) c_{2}^{2} b^{2}+\left(\frac{\lambda}{l}-1-\lambda\right) c_{1}^{2}+l c_{1} c_{2} b=-\lambda_{F} ; \\
-2(1-l) c_{1} c_{2} b^{2}+2 c_{1} c_{2}\left(\frac{\lambda}{l}-1-\lambda\right)+l\left(-c_{1}^{2}+c_{2}^{2}\right) b=0 .
\end{gathered}
$$

By (4.3i) and (4.3ii) and $b^{2}=\frac{\lambda}{l}-1$, we get

$$
\left[(1-l) b^{2}-\left(\frac{\lambda}{l}-1-\lambda\right)\right]\left(c_{1}^{2}-c_{2}^{2}\right)-2 l c_{1} c_{2} b=0, c_{1}^{2}-c_{2}^{2}=2 c_{1} c_{2} b .
$$

By (4.4) and (4.3iii), we have $c_{1} c_{2}=0$, then $c_{1}=c_{2}=0$. This is a contradiction.

Case iii) $\lambda=l$ and $f=c_{1}+c_{2} t$, by Corollary 4.1(3), we get

$$
\lambda_{F}+(1-l) c_{2}^{2}+\left(\frac{\lambda}{l}-1-\lambda\right)\left(c_{1}+c_{2} t\right)^{2}+l\left(c_{1}+c_{2} t\right) c_{2}=0 .
$$

Then

$$
\left(\frac{\lambda}{l}-1-\lambda\right) c_{2}^{2}=0
$$

$$
2\left(\frac{\lambda}{l}-1-\lambda\right) c_{1} c_{2}+l c_{2}^{2}=0
$$

$$
\lambda_{F}+(1-l) c_{2}^{2}+\left(\frac{\lambda}{l}-1-\lambda\right) c_{1}^{2}+l c_{1} c_{2}=0 .
$$

By $\lambda=l$ and (4.6i), then $c_{2}=0$. By (4.6iii), then $c_{1}=\sqrt{\frac{\lambda_{F}}{l}}$. So we get $\lambda=l, f=\sqrt{\frac{\lambda_{F}}{l}}$. We get the following theorem. 
Theorem 4.2. Let $M=I \times F$ with the metric tensor $-d t^{2}+f(t)^{2} g_{F}$ and $P=\frac{\partial}{\partial t}$ and $\operatorname{dim} F>1$. Then $(M, \bar{\nabla})$ is Einstein with the Einstein constant $\lambda$ if and only if $\left(F, \nabla^{F}\right)$ is Einstein with the Einstein constant $\lambda_{F}$ and one of the following conditions holds 1) $\left.\lambda_{F}=\lambda=0, f=c_{1} e^{t}, 2\right) \lambda=l, f=\sqrt{\frac{\lambda_{F}}{l}}$.

By (3.9) and Proposition 3.4, we have:

Corollary 4.3. Let $M=I \times F$ with the metric tensor $-d t^{2}+f(t)^{2} g_{F}$ and $P=\frac{\partial}{\partial t}$. If $(M, \bar{\nabla})$ has constant scalar curvature $\bar{S}$ if and only if $\left(F, \nabla^{F}\right)$ has constant scalar curvature $S^{F}$ and

$$
\bar{S}=\frac{S^{F}}{f^{2}}-2 l \frac{f^{\prime \prime}}{f}-l(l-1) \frac{f^{\prime 2}}{f^{2}}+l+l^{2} \frac{f^{\prime}}{f} .
$$

In (4.7), we make the change of variable $f(t)=\sqrt{v(t)}$ and have the following equation

$$
v^{\prime \prime}(t)+\frac{l-3}{4} \frac{v^{\prime}(t)^{2}}{v(t)}-\frac{l}{2} v^{\prime}(t)-\frac{l-\bar{S}}{l} v(t)-\frac{S^{F}}{l}=0 .
$$

Theorem 4.4. Let $M=I \times F$ with the metric tensor $-d t^{2}+f(t)^{2} g_{F}$ and $P=\frac{\partial}{\partial t}$ and $\operatorname{dim} F=l=3$. If $(M, \bar{\nabla})$ has constant scalar curvature $\bar{S}$ if and only if $\left(F, \nabla^{F}\right)$ has constant scalar curvature $S^{F}$ and

(1) $\bar{S}<\frac{75}{16}$ and $\bar{S} \neq 3, v(t)=c_{1} e^{\frac{\frac{3}{2}+\sqrt{\frac{25}{4}-\frac{4}{3} \bar{S}}}{2} t}+c_{2} e^{\frac{\frac{3}{2}-\sqrt{\frac{25}{4}-\frac{4}{3} \bar{S}}}{2} t}+\frac{S^{F}}{\bar{S}-3}$.

(2) $\bar{S}=\frac{75}{16}, v(t)=c_{1} e^{\frac{3}{4} t}+c_{2} t e^{\frac{3}{4} t}+\frac{S^{F}}{\bar{S}-3}$.

(3) $\bar{S}>\frac{75}{16}, v(t)=c_{1} e^{\frac{3}{4} t} \cos \left(\frac{\sqrt{\frac{3}{4} \bar{S}-\frac{25}{4}}}{2} t\right)+c_{2} e^{\frac{3}{4} t} \sin \left(\frac{\sqrt{\frac{3}{4} \bar{S}-\frac{25}{4}}}{2} t\right)+\frac{S^{F}}{\bar{S}-3}$.

(4) $\bar{S}=3, v(t)=c_{1}-\frac{2 S^{F}}{9} t+c_{2} e^{\frac{3}{2} t}$.

Proof. If $l=3$, then we have a simple differential equation

$$
v^{\prime \prime}(t)-\frac{3}{2} v^{\prime}(t)+\left(\frac{\bar{S}}{3}-1\right) v(t)-\frac{S^{F}}{3}=0 .
$$

If $\bar{S} \neq 3$, we putting $h(t)=\left(\frac{\bar{S}}{3}-1\right) v(t)-\frac{S^{F}}{3}$, it follows that $h^{\prime \prime}(t)-\frac{3}{2} h^{\prime}(t)+$ $\left(\frac{\bar{S}}{3}-1\right) h(t)=0$. The above solutions (1)-(3) follow directly from elementary methods for ordinary differential equations. When $\bar{S}=3$, then $v^{\prime \prime}(t)-\frac{3}{2} v^{\prime}(t)-$ $\frac{S^{F}}{3}=0$, we get the solution (4).

Theorem 4.5. Let $M=I \times F$ with the metric tensor $-d t^{2}+f(t)^{2} g_{F}$ and $P=\frac{\partial}{\partial t}$ and $\operatorname{dim} F=l \neq 3$ and $S^{F}=0$. Let $\triangle=\frac{l^{2}}{4}+\frac{(l+1)(l-\bar{S})}{l}$. If $(M, \bar{\nabla})$ has constant scalar curvature $\bar{S}$ if and only if
(1) $\bar{S}<\frac{l^{3}}{4(l+1)}+l, v(t)=\left(c_{1} e^{\frac{\frac{l}{2}+\sqrt{\Delta}}{2} t}+c_{2} e^{\frac{\frac{l}{2}-\sqrt{\square}}{2} t}\right)^{\frac{4}{l+1}}$.
(2) $\bar{S}=\frac{l^{3}}{4(l+1)}+l, v(t)=\left(c_{1} e^{\frac{l}{4} t}+c_{2} t e^{\frac{l}{4} t}\right)^{\frac{4}{l+1}}$. 
(3) $\bar{S}>\frac{l^{3}}{4(l+1)}+l, v(t)=\left(c_{1} e^{\frac{l}{4} t} \cos \left(\frac{\sqrt{-\triangle}}{2} t\right)+c_{2} e^{\frac{l}{4} t} \sin \left(\frac{\sqrt{-\triangle}}{2} t\right)\right)^{\frac{4}{l+1}}$.

Proof. In this case, the equation (4.8) is changed into the simpler form

$$
\frac{v^{\prime \prime}(t)}{v(t)}+\frac{l-3}{4} \frac{v^{\prime}(t)^{2}}{v(t)^{2}}-\frac{l}{2} \frac{v^{\prime}(t)}{v(t)}-\frac{l-\bar{S}}{l}=0 .
$$

Putting $v(t)=w(t)^{\frac{4}{l+1}}$, then $w(t)$ satisfies the equation $w^{\prime \prime}-\frac{l}{2} w^{\prime}+\frac{(l+1)}{4} \frac{(\bar{S}-l)}{l} w$ $=0$, by the elementary methods for ordinary differential equations, we prove the above theorem.

When $\operatorname{dim} F=l \neq 3$ and $S^{F} \neq 0$, putting $v(t)=w(t)^{\frac{4}{t+1}}$, then $w(t)$ satisfies the equation

$$
w^{\prime \prime}-\frac{l}{2} w^{\prime}+\frac{(l+1)}{4} \frac{(\bar{S}-l)}{l} w-\frac{(l+1)}{4} \frac{S^{F}}{l} w^{1-\frac{4}{l+1}}=0 .
$$

\section{Generalized Kasner spacetimes with a semi-symmetric non-metric connection}

In this section, we consider the scalar and Ricci curvature of generalized Kasner spacetimes with a semi-symmetric non-metric connection. We recall the definition of generalized Kasner spacetimes ([6]).

Definition 5.1. A generalized Kasner spacetime $(M, g)$ is a Lorentzian multiply warped product of the form $M=I \times_{\phi^{p_{1}}} F_{1} \times \cdots \times_{\phi^{p_{m}}} F_{m}$ with the metric $g=-d t^{2} \oplus \phi^{2 p_{1}} g_{F_{1}} \oplus \cdots \oplus \phi^{2 p_{m}} g_{F_{m}}$, where $\phi: I \rightarrow(0, \infty)$ is smooth and $p_{i} \in \mathbb{R}$ for any $i \in\{1, \ldots, m\}$ and also $I=\left(t_{1}, t_{2}\right)$.

We introduce the following parameters $\zeta=\sum_{i=1}^{m} l_{i} p_{i}$ and $\eta=\sum_{i=1}^{m} l_{i} p_{i}^{2}$ for generalized Kasner spacetimes. By Theorem 3.1 and direct computations, we get:

Proposition 5.2. Let $M=I \times_{\phi^{p_{1}}} F_{1} \times \cdots \times_{\phi^{p_{m}}} F_{m}$ be a generalized Kasner spacetime and $P=\frac{\partial}{\partial t}$. Then $(M, \bar{\nabla})$ is Einstein with the Einstein constant $\lambda$ if and only if the following conditions are satisfied for any $i \in\{1, \ldots, m\}$

(1) $\left(F_{i}, \nabla^{F_{i}}\right)$ is Einstein with the Einstein constant $\lambda_{i}, i \in\{1, \ldots, m\}$.

(2) $(\eta-\zeta) \frac{\phi^{\prime 2}}{\phi^{2}}+\zeta\left(\frac{\phi^{\prime \prime}}{\phi}\right)+\lambda-\sum_{i=1}^{m} l_{i}=0$.

(3) $\frac{\lambda_{i}}{\phi^{2} p_{i}}-p_{i} \frac{\phi^{\prime \prime}}{\phi}-(\zeta-1) p_{i} \frac{\phi^{\prime 2}}{\phi^{2}}+\zeta \frac{\phi^{\prime}}{\phi}=\lambda$.

By (3.9) we obtain:

Proposition 5.3. Let $M=I \times_{\phi^{p_{1}}} F_{1} \times \cdots \times_{\phi^{p_{m}}} F_{m}$ be a generalized Kasner spacetime and $P=\frac{\partial}{\partial t}$. Then $(M, \bar{\nabla})$ has constant scalar curvature $\bar{S}$ if and only if each $\left(F_{i}, \nabla^{F_{i}}\right)$ has constant scalar curvature $S^{F_{i}}$ and

$$
\bar{S}=\sum_{i=1}^{m} \frac{S^{F_{i}}}{\phi^{2 p_{i}}}-2 \zeta \frac{\phi^{\prime \prime}}{\phi}-\left(\eta+\zeta^{2}-2 \zeta\right) \frac{\phi^{\prime 2}}{\phi^{2}}+(\bar{n}-1) \zeta \frac{\phi^{\prime}}{\phi}+(\bar{n}-1) .
$$


Nextly, we first give a classification of four-dimensional generalized Kasner spacetimes with a semi-symmetric non-metric connection and then consider Ricci tensors and scalar curvatures of them.

Definition 5.4. Let $M=I \times_{b_{1}} F_{1} \times \cdots \times_{b_{m}} F_{m}$ with the metric $g=-d t^{2} \oplus$ $b_{1}^{2} g_{F_{1}} \oplus \cdots \oplus b_{m}^{2} g_{F_{m}}$.

- $(M, g)$ is said to be of Type (I) if $m=1$ and $\operatorname{dim}(F)=3$.

- $(M, g)$ is said to be of Type (II) if $m=2$ and $\operatorname{dim}\left(F_{1}\right)=1$ and $\operatorname{dim}\left(F_{2}\right)=2$.

- $(M, g)$ is said to be of Type (III) if $m=3$ and $\operatorname{dim}\left(F_{1}\right)=1, \operatorname{dim}\left(F_{2}\right)=1$ and $\operatorname{dim}\left(F_{3}\right)=1$.

By Theorems 4.2 and 4.4, we have given a classification of Type (I) Einstein spaces and Type (I) spaces with the constant scalar curvature.

- Classification of Einstein Type (II) generalized Kasner spacetimes with a semi-symmetric non-metric connection

Let $M=I \times_{\phi^{p_{1}}} F_{1} \times_{\phi^{p_{2}}} F_{2}$ be an Einstein type (II) generalized Kasner spacetime and $P=\frac{\partial}{\partial t}$. Then $\zeta=p_{1}+2 p_{2}, \eta=p_{1}^{2}+2 p_{2}^{2}$. By Proposition 5.2, we have

$$
\begin{gathered}
(\eta-\zeta) \frac{\phi^{2}}{\phi^{2}}+\zeta\left(\frac{\phi^{\prime \prime}}{\phi}\right)+\lambda-3=0 ; \\
-p_{1} \frac{\phi^{\prime \prime}}{\phi}-(\zeta-1) p_{1} \frac{\phi^{2}}{\phi^{2}}+\zeta \frac{\phi^{\prime}}{\phi}=\lambda ; \\
\frac{\lambda_{2}}{\phi^{2 p_{2}}}-p_{2} \frac{\phi^{\prime \prime}}{\phi}-(\zeta-1) p_{2} \frac{\phi^{\prime 2}}{\phi^{2}}+\zeta \frac{\phi^{\prime}}{\phi}=\lambda,
\end{gathered}
$$

where $\lambda_{2}$ is a constant. Consider following two cases:

Case i) $\zeta=0$.

In this case, $p_{2}=-\frac{1}{2} p_{1}, \eta=\frac{3}{2} p_{1}^{2}$. Then by (5.2), we have

$$
\begin{gathered}
\eta \frac{\phi^{\prime 2}}{\phi^{2}}+\lambda-3=0, \\
p_{1}\left(-\frac{\phi^{\prime \prime}}{\phi}+\frac{\phi^{2}}{\phi^{2}}\right)=\lambda, \\
\frac{\lambda_{2}}{\phi^{-p_{1}}}-\frac{1}{2} p_{1}\left(-\frac{\phi^{\prime \prime}}{\phi}+\frac{\phi^{2}}{\phi^{2}}\right)=\lambda .
\end{gathered}
$$

Case i a) $\eta=0$, then $p_{i}=0$, by (5.3i), $\lambda=3$. By (5.3ii), $\lambda=0$, this is a contradiction.

Case i b) $\eta \neq 0$, then $p_{i} \neq 0$.

Case i b)1) $\underline{\lambda_{2}}=0$. By (5.3ii) and (5.3iii), $\lambda=0$ and

$$
-\frac{\phi^{\prime \prime}}{\phi}+\frac{\phi^{\prime 2}}{\phi^{2}}=0, \frac{\phi^{2}}{\phi^{2}}=\frac{3}{\eta},
$$


then $\phi=c_{0} e^{ \pm \sqrt{\frac{3}{\eta}} t}$ which satisfies the first equation in (5.4), so we get

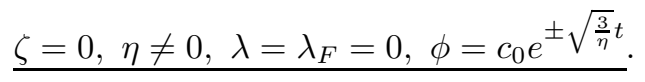

Case i b)2) $\underline{\lambda_{2} \neq 0}$. By (5.3ii) and (5.3iii), we have $\frac{\lambda_{2}}{\phi^{-p_{1}}}=\frac{3}{2} \lambda$, so $\phi$ is a constant. By (5.3ii), $\lambda=0$, so $\lambda_{2}=0$, this is a contradiction.

Case ii) $\underline{\zeta \neq 0}$, then $\eta \neq 0$. Putting $\phi=\psi^{\frac{\zeta}{\eta}}$, then $\psi^{\prime \prime}+\frac{(\lambda-3) \eta}{\zeta^{2}} \psi=0$. Hence,

(1) $\lambda<3, \psi=c_{1} e^{\sqrt{\frac{(3-\lambda) \eta}{\zeta^{2}}} t}+c_{2} e^{-\sqrt{\frac{(3-\lambda) \eta}{\zeta^{2}}} t}$,

(2) $\lambda=3, \psi=c_{1}+c_{2} t$,

(3) $\lambda>3, \psi=c_{1} \cos \left(\sqrt{\frac{(\lambda-3) \eta}{\zeta^{2}}} t\right)+c_{2} \sin \left(\sqrt{\frac{(\lambda-3) \eta}{\zeta^{2}}} t\right)$.

We make (5.2) into

$$
\begin{gathered}
\psi^{\prime \prime}+\frac{(\lambda-3) \eta}{\zeta^{2}} \psi=0 \\
-\frac{p_{1}}{\zeta} \frac{\left(\phi^{\zeta}\right)^{\prime \prime}}{\phi^{\zeta}}+\frac{\left(\phi^{\zeta}\right)^{\prime}}{\phi^{\zeta}}=\lambda ; \\
\frac{\lambda_{2}}{\phi^{2 p_{2}}}-\frac{p_{2}}{\zeta} \frac{\left(\phi^{\zeta}\right)^{\prime \prime}}{\phi^{\zeta}}+\frac{\left(\phi^{\zeta}\right)^{\prime}}{\phi^{\zeta}}=\lambda .
\end{gathered}
$$

When $p_{1}=p_{2}$, the type (II) spaces turns into type (I) spaces, so we assume $p_{1} \neq p_{2}$. By (5.5ii) and (5.5iii), then

$$
\psi^{\prime}=\frac{p_{1} \lambda_{2} \eta}{\left(p_{2}-p_{1}\right) \zeta^{2}} \psi^{1-\frac{2 p_{2} \zeta}{\eta}}+\frac{\lambda \eta}{\zeta^{2}} \psi
$$

Case ii)(1) $\lambda<3, \psi=c_{1} e^{a t}+c_{2} e^{-a t}$, where $a=\sqrt{\frac{(3-\lambda) \eta}{\zeta^{2}}}$. By (5.6),

$$
a c_{1} e^{a t}-a c_{2} e^{-a t}=\frac{p_{1} \lambda_{2} \eta}{\left(p_{2}-p_{1}\right) \zeta^{2}}\left(c_{1} e^{a t}+c_{2} e^{-a t}\right)^{1-\frac{2 p_{2} \zeta}{\eta}}+\frac{\lambda \eta}{\zeta^{2}}\left(c_{1} e^{a t}+c_{2} e^{-a t}\right) .
$$

Case ii)(1)(a) $\underline{c_{1}=0}$, then

$$
-\left[a+\frac{\lambda \eta}{\zeta^{2}}\right] c_{2} e^{-a t}=\frac{p_{1} \lambda_{2} \eta}{\left(p_{2}-p_{1}\right) \zeta^{2}}\left(c_{2} e^{-a t}\right)^{1-\frac{2 p_{2} \zeta}{\eta}} .
$$

Case ii)(1)(a)1) $\underline{p_{1} \lambda_{2} \neq 0}$, then $p_{2}=0$ and $\zeta=p_{1}, \eta=p_{1}^{2}$ and $\psi=c_{2} e^{-a t}$. By (5.5ii), we get $-\overline{a^{2}-a}=\lambda$ and $-\sqrt{3-\lambda}=3$, this is a contradiction.

Case ii)(1)(a)2) $\underline{p_{1} \lambda_{2}=0}$. If $\underline{p_{1}=0}$, then $\zeta=2 p_{2}, \eta=2 p_{2}^{2}$. By (5.8), $a=-\frac{\lambda}{2}$ and $\phi^{\zeta}=c_{2} e^{-2 a t}$. By (5.5iii), then

$$
\frac{\lambda_{2}}{c^{\prime} e^{\frac{-4 p_{2} a t}{\zeta}}}-2 a^{2}-2 a=\lambda
$$


so $\lambda_{2}=0$ and $-2 a^{2}-2 a=\lambda$. By $a=-\frac{\lambda}{2}$, then $\lambda=0$ and $a=0$, this is a contradiction.

If $\underline{\lambda_{2}=0, p_{1} \neq 0}$, by (5.5ii) and $a=-\frac{\lambda \eta}{\zeta^{2}}$ and $\phi^{\zeta}=c^{\prime} e^{-\frac{a t \zeta^{2}}{\eta}}$, then $\lambda=0$ and $a=0$, this is a contradiction.

Case ii)(1)(b) $\underline{c_{2}=0}$, then

$$
\left[a-\frac{\lambda \eta}{\zeta^{2}}\right] c_{1} e^{a t}=\frac{p_{1} \lambda_{2} \eta}{\left(p_{2}-p_{1}\right) \zeta^{2}}\left(c_{1} e^{a t}\right)^{1-\frac{2 p_{2} \zeta}{\eta}} .
$$

Case ii)(1)(b)1) $p_{1} \lambda_{2} \neq 0$, then $p_{2}=0$ and $\zeta=p_{1}, \eta=p_{1}^{2}$ and $\sqrt{3-\lambda}-\lambda=$ $-\lambda_{2}$. By (5.5ii), we get $-a^{2}+a=\lambda$ and $\lambda=-6$, so $\lambda_{2}=-9$. In this case, (5.2iii) holds, so we get $p_{2}=0, p_{1} \neq 0, \lambda=-6, \lambda_{2}=-9, \phi=c_{1} e^{\frac{3 t}{\zeta}}$.

Case ii)(1)(b)2) $\underline{p_{1} \lambda_{2}}=0$. If $\underline{p_{1}=0}$, then $\zeta=2 p_{2}, \eta=2 p_{2}^{2}$ and $\psi=c_{1} e^{a t}$ and $a=\frac{\lambda \eta}{\zeta^{2}}=\frac{\lambda}{2}$. By (5.5iii),

$$
\frac{\lambda_{2}}{c^{\prime} e^{\frac{4 p_{2} a t}{\zeta}}}-2 a^{2}+2 a=\lambda,
$$

so $\lambda_{2}=0$ and $-2 a^{2}+2 a=\lambda$, then $\lambda=a=0$, this is a contradiction. If $\lambda_{2}=0$, by (5.5ii), then $\lambda=a=0$, this is a contradiction.

Case ii)(1)(c) $\underline{c_{1} \neq 0, c_{2} \neq 0}$.

If $\underline{p_{2} \neq 0}$, then $e^{a t}, e^{-a t},\left(c_{1} e^{a t}+c_{2} e^{-a t}\right)^{1-\frac{2 p_{2} \zeta}{\eta}}$ are linear independent, by (5.7), then

$$
\left[a-\frac{\lambda \eta}{\zeta^{2}}\right] c_{1}=0,\left[-a-\frac{\lambda \eta}{\zeta^{2}}\right] c_{2}=0, \frac{p_{1} \lambda_{2} \eta}{\left(p_{2}-p_{1}\right) \zeta^{2}}=0 .
$$

So $a=0$, this is a contradiction.

If $p_{2}=0$, then by $(5.7)$,

$$
a-\frac{\lambda \eta}{\zeta^{2}}-\frac{p_{1} \lambda_{2} \eta}{\left(p_{2}-p_{1}\right) \zeta^{2}}=0,-a-\frac{\lambda \eta}{\zeta^{2}}-\frac{p_{1} \lambda_{2} \eta}{\left(p_{2}-p_{1}\right) \zeta^{2}}=0,
$$

so $a=0$ and we get a contradiction.

Case ii)(2) $\lambda=3, \psi=c_{1}+c_{2} t$. By (5.6), we have

$$
c_{2}=\frac{p_{1} \lambda_{2} \eta}{\left(p_{2}-p_{1}\right) \zeta^{2}}\left(c_{1}+c_{2} t\right)^{1-\frac{2 p_{2} \zeta}{\eta}}+\frac{3 \eta}{\zeta^{2}}\left(c_{1}+c_{2} t\right) .
$$

Case ii)(2)a) $c_{2} \neq 0$, so $p_{2}=0$. By (5.5iii), then $\phi^{\zeta}=c_{0} e^{\left(-\lambda_{2}+\lambda\right) t}$ and $c_{1}+c_{2} t=c_{0} e^{\left(-\overline{\left.\lambda_{2}+\lambda\right) t}\right.}$, this is a contradiction with $c_{2} \neq 0$.

Case ii)(2)b) $c_{2}=0$, then $\psi$ and $\phi$ are constants, by (5.5ii), then $\lambda=0$. This is a contradiction with $\lambda=3$.

Case ii)(3) $\lambda>3, \psi=c_{1} \cos (a t)+c_{2} \sin (a t)$, where $a=\sqrt{\frac{(\lambda-3) \eta}{\zeta^{2}}}$. By (5.6), we have

$$
a\left(-c_{1} \sin (a t)+c_{2} \cos (a t)\right)=\frac{p_{1} \lambda_{2} \eta}{\left(p_{2}-p_{1}\right) \zeta^{2}}\left(c_{1} \cos (a t)+c_{2} \sin (a t)\right)^{1-\frac{2 p_{2} \zeta}{\eta}}
$$




$$
+\frac{\lambda \eta}{\zeta^{2}}\left(c_{1} \cos (a t)+c_{2} \sin (a t)\right) .
$$

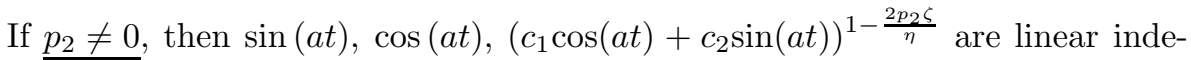
pendent, so $-a c_{1}=\frac{\lambda \eta}{\zeta^{2}} c_{2}, a c_{2}=\frac{\lambda \eta}{\zeta^{2}} c_{1}$ and $a=0$, this is a contradiction. If $p_{2}=0$, then

$$
-a c_{1}=\frac{p_{1} \lambda_{2} \eta}{\left(p_{2}-p_{1}\right) \zeta^{2}} c_{2}+\frac{\lambda \eta}{\zeta^{2}} c_{2} ; a c_{2}=\frac{p_{1} \lambda_{2} \eta}{\left(p_{2}-p_{1}\right) \zeta^{2}} c_{1}+\frac{\lambda \eta}{\zeta^{2}} c_{1} .
$$

Then $a=0$, this is a contradiction. By the above discussions, we get the following theorem:

Theorem 5.5. Let $M=I \times_{\phi^{p_{1}}} F_{1} \times_{\phi^{p_{2}}} F_{2}$ be a generalized Kasner spacetime and $\operatorname{dim} F_{1}=1, \operatorname{dim} F_{2}=2$ and $P=\frac{\partial}{\partial t}$. Then $(M, \bar{\nabla})$ is Einstein with the Einstein constant $\lambda$ if and only if $\left(F_{2}, \nabla^{F_{2}}\right)$ is Einstein with the Einstein constant $\lambda_{2}$, and one of the following conditions is satisfied

(1) $\zeta=0, \eta \neq 0, \lambda=\lambda_{F}=0, \phi=c_{0} e^{ \pm \sqrt{\frac{3}{\eta}} t}$.

(2) $p_{2}=0, p_{1} \neq 0, \lambda=-6, \lambda_{2}=-9, \phi=c_{1} e^{\frac{3 t}{\zeta}}$.

- Type (II) generalized Kasner space-times with a semi-symmetric non-metric connection with constant scalar curvature

By Proposition 5.3, then $\left(F_{2}, \nabla^{F_{2}}\right)$ has constant scalar curvature $S^{F_{2}}$ and

$$
\bar{S}=\frac{S^{F_{2}}}{\phi^{2 p_{2}}}-2 \zeta \frac{\phi^{\prime \prime}}{\phi}-\left(\eta+\zeta^{2}-2 \zeta\right) \frac{\phi^{2}}{\phi^{2}}+3 \zeta \frac{\phi^{\prime}}{\phi}+3 .
$$

If $\underline{\zeta=0}$, when $\underline{\eta=0}$, then $p_{1}=p_{2}=0$ and $\underline{\bar{S}=S^{F_{2}}+3}$. If $\underline{\eta \neq 0}$, then

$$
\eta \frac{\phi^{\prime 2}}{\phi^{2}}=\frac{S^{F_{2}}}{\phi^{2 p_{2}}}+(-\bar{S}+3) .
$$

If $\underline{\zeta \neq 0}$, putting $\phi=\psi^{\frac{2 \zeta}{\eta+\zeta^{2}}}$, we get

$$
-\frac{4 \zeta^{2}}{\eta+\zeta^{2}} \psi^{\prime \prime}+\frac{6 \zeta^{2}}{\eta+\zeta^{2}} \psi^{\prime}+(-\bar{S}+3) \psi+S^{F_{2}} \psi^{1-\frac{4 p_{2} \zeta}{\eta+\zeta^{2}}}=0 .
$$

- Type (III) generalized Kasner space-times with a semi-symmetric non-metric connection with constant scalar curvature

By Proposition 5.3, then

$$
\bar{S}=-2 \zeta \frac{\phi^{\prime \prime}}{\phi}-\left(\eta+\zeta^{2}-2 \zeta\right) \frac{\phi^{2}}{\phi^{2}}+3 \zeta \frac{\phi^{\prime}}{\phi}+3 .
$$

If $\underline{\zeta=\eta=0}$, then $p_{1}=p_{2}=p_{3}=0$, we get $\bar{S}=3$.

If $\underline{\zeta=0, \eta \neq 0}$, then $\left[(\ln \phi)^{\prime}\right]^{2}=-\frac{\bar{S}-3}{\eta}$, so when $\bar{S}>3$, there is no solutions, 
when $\bar{S}=3, \phi$ is a constant and when $\bar{S}<3, \phi=c_{0} e^{ \pm \sqrt{\frac{-\bar{S}+3}{\eta}} t}$. If $\zeta \neq 0$, then $\eta \neq 0$, putting $\phi=\psi^{\frac{2 \zeta}{\eta+\zeta^{2}}}$, then

$$
\psi^{\prime \prime}-\frac{3}{2} \psi^{\prime}+\frac{(\bar{S}-3)\left(\eta+\zeta^{2}\right)}{4 \zeta^{2}} \psi=0 .
$$

Let $\triangle=\frac{9}{4}-\frac{(\bar{S}-3)\left(\eta+\zeta^{2}\right)}{\zeta^{2}}$. So we get

(1) $\bar{S}<\frac{9 \zeta^{2}}{4\left(\eta+\zeta^{2}\right)}+3, \psi=c_{1} e^{\frac{\frac{3}{2}+\sqrt{\Delta}}{2} t}+c_{2} e^{\frac{\frac{3}{2}-\sqrt{\Delta}}{2} t}$,

(2) $\bar{S}=\frac{9 \zeta^{2}}{4\left(\eta+\zeta^{2}\right)}+3, \psi=c_{1} e^{\frac{3}{4} t}+c_{2} t e^{\frac{3}{4} t}$,

(3) $\bar{S}>\frac{9 \zeta^{2}}{4\left(\eta+\zeta^{2}\right)}+3, \psi=c_{1} e^{\frac{3}{4} t} \cos \left(\frac{\sqrt{-\triangle}}{2} t\right)+c_{2} e^{\frac{3}{4} t} \sin \left(\frac{\sqrt{-\triangle}}{2} t\right)$. So we get the following theorem.

Theorem 5.6. Let $M=I \times_{\phi^{p_{1}}} F_{1} \times_{\phi^{p_{2}}} F_{2} \times_{\phi^{p_{3}}} F_{3}$ be a generalized Kasner spacetime and $\operatorname{dim} F_{1}=\operatorname{dim} F_{2}=\operatorname{dim} F_{3}=1$, and $P=\frac{\partial}{\partial t}$. Then $\bar{S}$ is a constant if and only if one of the following case holds

(1) $\zeta=\eta=0, \bar{S}=3$.

(2) $\zeta=0, \eta \neq 0$, when $\bar{S}>3$, there is no solutions, when $\bar{S}=3, \phi$ is a constant and when $\bar{S}<3, \phi=c_{0} e^{ \pm \sqrt{-\frac{\bar{S}-3}{\eta}} t}$.

(3) If $\zeta \neq 0$.

(3a) $\bar{S}<\frac{9 \zeta^{2}}{4\left(\eta+\zeta^{2}\right)}+3, \phi=\left(c_{1} e^{\frac{\frac{3}{2}+\sqrt{\Xi}}{2} t}+c_{2} e^{\frac{\frac{3}{2}-\sqrt{\Xi}}{2} t}\right)^{\frac{2 \zeta}{\eta+\zeta^{2}}}$,

(3b) $\bar{S}=\frac{9 \zeta^{2}}{4\left(\eta+\zeta^{2}\right)}+3, \phi=\left(c_{1} e^{\frac{3}{4} t}+c_{2} t e^{\frac{3}{4} t}\right)^{\frac{2 \zeta}{\eta+\zeta^{2}}}$,

(3c) $\bar{S}>\frac{9 \zeta^{2}}{4\left(\eta+\zeta^{2}\right)}+3, \phi=\left(c_{1} e^{\frac{3}{4} t} \cos \left(\frac{\sqrt{-\triangle}}{2} t\right)+c_{2} e^{\frac{3}{4} t} \sin \left(\frac{\sqrt{-\triangle}}{2} t\right)\right)^{\frac{2 \zeta}{\eta+\zeta^{2}}}$.

- Einstein Type (III) generalized Kasner space-times with a semisymmetric non-metric connection.

By Proposition 5.2, we have

$$
\begin{aligned}
& \zeta\left(\frac{\phi^{\prime \prime}}{\phi}\right)+(\eta-\zeta) \frac{\phi^{2}}{\phi^{2}}+\lambda-3=0, \\
& -p_{1}\left[\frac{\phi^{\prime \prime}}{\phi}+(\zeta-1) \frac{\phi^{\prime 2}}{\phi^{2}}\right]+\zeta \frac{\phi^{\prime}}{\phi}=\lambda, \\
& -p_{2}\left[\frac{\phi^{\prime \prime}}{\phi}+(\zeta-1) \frac{\phi^{\prime 2}}{\phi^{2}}\right]+\zeta \frac{\phi^{\prime}}{\phi}=\lambda, \\
& -p_{3}\left[\frac{\phi^{\prime \prime}}{\phi}+(\zeta-1) \frac{\phi^{\prime 2}}{\phi^{2}}\right]+\zeta \frac{\phi^{\prime}}{\phi}=\lambda .
\end{aligned}
$$

If $\zeta=\eta=0$, by (5.22i), $\lambda=3$, by (4.50ii), $\lambda=0$, this is a contradiction.

If $\underline{\zeta=0, \eta \neq 0}$, plusing (5.22ii), (5.22iii), (5.22iv), we get $\lambda=0$. By (5.22i), 
$\frac{\phi^{\prime 2}}{\phi^{2}}=\frac{3}{\eta}$ and $\phi=c_{0} e^{ \pm \sqrt{\frac{3}{\eta}} t}$ which satisfies (5.22ii), (5.22iii) and (5.22iv), so we obtain $\lambda=0, \zeta=0, \eta \neq 0, \phi=c_{0} e^{ \pm \sqrt{\frac{3}{\eta}} t}$.

When $\underline{\zeta \neq 0}$, if $p_{1}=p_{2}=p_{3}$, we get type (I), so we may let $p_{1} \neq p_{2}$. By (5.22ii) and (5.22iii), we have $\frac{\left(\phi^{\zeta}\right)^{\prime}}{\phi^{\zeta}}=\lambda$ and $\frac{\left(\phi^{\zeta}\right)^{\prime \prime}}{\phi^{\zeta}}=0$, so $\phi^{\zeta}=c_{1}+c_{2} t$ and $c_{2}=\lambda\left(c_{1}+c_{2} t\right)$, then $\lambda c_{2}=0$. When $c_{2}=0$, then $\lambda=0$ and $\phi$ is a constant, so by $(5.22 \mathrm{i})$, then $\lambda=3$ which is a contradiction. When $\lambda=0$, then $c_{2}=0$ which is also a contradiction. We get the following theorem.

Theorem 5.7. Let $M=I \times_{\phi^{p_{1}}} F_{1} \times_{\phi^{p_{2}}} F_{2} \times_{\phi^{p_{3}}} F_{3}$ be a generalized Kasner spacetime for $p_{i} \neq p_{j}$ for some $i, j \in\{1,2,3\}$ and $\operatorname{dim} F_{1}=\operatorname{dim} F_{2}=\operatorname{dim} F_{3}=$ 1 , and $P=\frac{\partial}{\partial t}$. Then $(M, \bar{\nabla})$ is Einstein with the Einstein constant $\lambda$ if and only if $\lambda=0, \zeta=0, \eta \neq 0, \phi=c_{0} e^{ \pm \sqrt{\frac{3}{\eta}} t}$.

\section{Curvature of multiply twisted products with an affine connection with a zero torsion}

Let $\nabla$ is the Levi-Civita connection of $M$, we define

$$
\widetilde{\nabla}_{X} Y=\nabla_{X} Y+\pi(X) Y+\pi(Y) X,
$$

which has no torsion. Let $R$ and $\widetilde{R}$ be the curvature tensors of $\nabla$ and $\widetilde{\nabla}$, respectively. Then $R$ and $\widetilde{R}$ are related by

$$
\begin{aligned}
\widetilde{R}(X, Y) Z= & R(X, Y) Z+X(\pi(Y)) Z-Y(\pi(X)) Z \\
& +g\left(Z, \nabla_{X} P\right) Y-g\left(Z, \nabla_{Y} P\right) X+\pi(Z)[\pi(Y) X-\pi(X) Y] \\
& -\pi([X, Y]) Z \\
= & \bar{R}(X, Y) Z+X(\pi(Y)) Z-Y(\pi(X)) Z-\pi([X, Y]) Z
\end{aligned}
$$

for any vector fields $X, Y, Z$ on $M$. By (6.1) and Proposition 2.2 in [12], we have:

Proposition 6.1. Let $M=B \times_{b_{1}} F_{1} \times_{b_{2}} F_{2} \times \cdots \times_{b_{m}} F_{m}$ be a multiply twisted product and let $X, Y \in \Gamma(T B)$ and $U \in \Gamma\left(T F_{i}\right), W \in \Gamma\left(T F_{j}\right)$ and $P \in \Gamma(T B)$. Then

(1) $\widetilde{\nabla}_{X} Y=\widetilde{\nabla}_{X}^{B} Y$.

(2) $\widetilde{\nabla}_{X} U=\left[\frac{X\left(b_{i}\right)}{b_{i}}+\pi(X)\right] U$.

(3) $\widetilde{\nabla}_{U} X=\left[\frac{X\left(b_{i}\right)}{b_{i}}+\pi(X)\right] U$.

(4) $\widetilde{\nabla}_{U} W=0$ if $i \neq j$.

(5) $\widetilde{\nabla}_{U} W=U\left(\ln b_{i}\right) W+W\left(\ln b_{i}\right) U-\frac{g_{F_{i}}(U, W)}{b_{i}} \operatorname{grad}_{F_{i}} b_{i}-b_{i} g_{F_{i}}(U, W) \operatorname{grad}_{B} b_{i}$ $+\nabla_{U}^{F_{i}} W$ if $i=j$.

Proposition 6.2. Let $M=B \times_{b_{1}} F_{1} \times_{b_{2}} F_{2} \times \cdots \times_{b_{m}} F_{m}$ be a multiply twisted product and let $X, Y \in \Gamma(T B)$ and $U \in \Gamma\left(T F_{i}\right), W \in \Gamma\left(T F_{j}\right)$ and $P \in \Gamma\left(T F_{k}\right)$. Then 
(1) $\widetilde{\nabla}_{X} Y=\nabla_{X}^{B} Y$.

(2) $\widetilde{\nabla}_{X} U=\frac{X\left(b_{i}\right)}{b_{i}} U+g(P, U) X$.

(3) $\widetilde{\nabla}_{U} X=\frac{X\left(b_{i}\right)}{b_{i}} U+g(P, U) X$.

(4) $\widetilde{\nabla}_{U} W=\pi(W) U+\pi(U) W$ if $i \neq j$.

(5) $\widetilde{\nabla}_{U} W=U\left(\ln b_{i}\right) W+W\left(\ln b_{i}\right) U-\frac{g_{F_{i}}(U, W)}{b_{i}} \operatorname{grad}_{F_{i}} b_{i}-b_{i} g_{F_{i}}(U, W) \operatorname{grad}_{B} b_{i}$ $+\nabla_{U}^{F_{i}} W+\pi(W) U+\pi(U) W$ if $i=j$.

By (6.2) and Propositions 2.4 and 2.5, we have:

Proposition 6.3. Let $M=B \times_{b_{1}} F_{1} \times_{b_{2}} F_{2} \times \cdots \times_{b_{m}} F_{m}$ be a multiply twisted product and let $X, Y, Z \in \Gamma(T B)$ and $V \in \Gamma\left(T F_{i}\right), W \in \Gamma\left(T F_{j}\right), U \in \Gamma\left(T F_{k}\right)$ and $P \in \Gamma(T B)$. Then

(1) $\widetilde{R}(X, Y) Z=\widetilde{R}^{B}(X, Y) Z$,

(2) $\widetilde{R}(X, Y) V=X(\pi(Y)) V-Y(\pi(X)) V-\pi([X, Y]) V$, and for other components, $\widetilde{R}$ equals to $\bar{R}$.

Proposition 6.4. Let $M=B \times_{b_{1}} F_{1} \times_{b_{2}} F_{2} \times \cdots \times_{b_{m}} F_{m}$ be a multiply twisted product and let $X, Y, Z \in \Gamma(T B)$ and $V \in \Gamma\left(T F_{i}\right), W \in \Gamma\left(T F_{j}\right), U \in \Gamma\left(T F_{k}\right)$ and $P \in \Gamma\left(T F_{l}\right)$. Then

(1) $\widetilde{R}(V, W) X=\bar{R}(V, W) X+V(\pi(W)) X-W(\pi(V)) X-\pi([V, W]) X$.

(2) $\widetilde{R}(U, V) W=U(\pi(V)) W-V(\pi(U)) W-\pi([U, V]) W$ if $i=k \neq j$.

(3) $\bar{R}(U, V) W=g(U, W) \operatorname{grad}_{B}\left(V\left(\ln b_{i}\right)\right)-g(V, W) \operatorname{grad}_{B}\left(U\left(\ln b_{i}\right)\right)+R^{F_{i}}(U$, $V) W-\frac{\left.\operatorname{lgrad}_{B} b_{i}\right|_{B} ^{2}}{b_{i}^{2}}(g(V, W) U-g(U, W) V)+g\left(W, \nabla_{U} P\right) V-g\left(W, \nabla_{V} P\right) U$ $+\pi(W)[\pi(V) U-\pi(U) V]+U(\pi(V)) W-V(\pi(U)) W-\pi([U, V]) W$ if $i=j=k=l$, and for other components, $\widetilde{R}$ equals to $\bar{R}$.

By Propositions 6.3 and 6.4, we have:

Proposition 6.5. Let $M=B \times_{b_{1}} F_{1} \times_{b_{2}} F_{2} \times \cdots \times_{b_{m}} F_{m}$ be a multiply twisted product and $P \in \Gamma(T B)$. Then $\widetilde{\text { Ric }}=\overline{\text { Ric. }}$

Remark. By Proposition 6.5, then we can get the same conclusions for $\widetilde{\nabla}$ and $\bar{\nabla}$.

Proposition 6.6. Let $M=B \times_{b_{1}} F_{1} \times_{b_{2}} F_{2} \times \cdots \times_{b_{m}} F_{m}$ be a multiply twisted product and $P \in \Gamma\left(T F_{r}\right)$. Then

$$
\begin{aligned}
\widetilde{\operatorname{Ric}}(V, W)= & \overline{\operatorname{Ric}}(V, W) \\
& +\sum_{j_{r}=1}^{l_{r}} \varepsilon_{j_{r}} g\left(V\left(\pi\left(E_{j_{r}}^{r}\right)\right) W-E_{j_{r}}^{r}(\pi(V)) W-\pi\left(\left[V, E_{j_{r}}^{r}\right]\right) W, E_{j_{r}}^{r}\right)
\end{aligned}
$$

for $V, W \in \Gamma\left(T F_{r}\right)$. For other components, $\widetilde{\text { Ric }}=\overline{\text { Ric. }}$

Acknowledgement. This work was supported by Fok Ying Tong Education Foundation No.121003 and NSFC No.11271062. I would like to thank the referee for his(her) careful reading and helpful comments. 


\section{References}

[1] N. Agashe and M. Chafle, A semi-symmetric nonmetric connection on a Riemannian manifold, Indian J. Pure Appl. Math. 23 (1992), 399-409.

[2] — On submanifolds of a Riemannian manifold with a semi-symmetric non-metric connection, Tensor (N.S.) 55 (1994), no. 2, 120-130.

[3] L. Alías, A. Romero, and M. Sánchez, Spacelike hypersurfaces of constant mean curvature and Calabi-Bernstein type problems, Tohoku Math. J. (2) 49 (1997), no. 3, 337-345.

[4] R. Bishop and B. O'Neill, Manifolds of negative curvature, Trans. Am. Math. Soc. 145 (1969), 1-49.

5] F. Dobarro and E. Dozo, Scalar curvature and warped products of Riemann manifolds, Trans. Amer. Math. Soc. 303 (1987), no. 1, 161-168.

[6] F. Dobarro and B. Ünal, Curvature of multiply warped products, J. Geom. Phys. 55 (2005), no. 1, 75-106.

[7] P. Ehrlich, Y. Jung, and S. Kim, Constant scalar curvatures on warped product manifolds, Tsukuba J. Math. 20 (1996), no. 1, 239-265.

[8] M. Fernández-López, E. García-Río, D. Kupeli, and B. Ünal, A curvature condition for a twisted product to be a warped product, Manuscripta Math. 106 (2001), no. 2, 213-217.

[9] H. Hayden, Subspace of a space with torsion, Proc. Lond. Math. Soc. 34 (1932), 27-50.

[10] C. Özgur and S. Sular, Warped products with a semi-symmetric non-metric connection, Arab. J. Sci. Eng. 36 (2011), no. 3, 461-473.

[11] S. Sular and C. Özgur, Warped products with a semi-symmetric metric connection, Taiwanese J. Math. 15 (2011), no. 4, 1701-1719.

[12] Y. Wang, Multiply twisted products, arXiv:1207.0199.

[13] K. Yano, On semi-symmetric metric connection, Rev. Roumaine Math. Pures Appl. 15 (1970), 1579-1586

School of Mathematics and Statistics

Northeast Normal University

Changchun Jilin, 130024, P. R. China

E-mail address: wangy581@nenu.edu.cn 